\title{
EFFECTS OF LINEAR FILTER ON STABILITY AND PERFORMANCE OF HUMAN-IN-THE-LOOP MODEL REFERENCE ADAPTIVE CONTROL ARCHITECTURES
}

\author{
Ehsan Yousefi \\ Mechanical Engineering \\ Bilkent University \\ Ankara, 06800, Turkey \\ Email: ehsan.yousefi@bilkent.edu.tr
}

\author{
Didem Fatma Demir \\ Mechanical Engineering \\ Bilkent University \\ Ankara, 06800, Turkey \\ Email: didem.demir@ug.bilkent.edu.tr
}

\author{
Rifat Sipahi \\ Mechanical and Industrial Engineering \\ Northeastern University \\ Boston, Massachusetts, 02115, USA \\ Email: rifat@coe.neu.edu
}

\author{
Tansel Yucelen \\ Mechanical Engineering \\ University of South Florida \\ Tampa, Florida, 33620, USA \\ Email: yucelen@lacis.team
}

\author{
Yildiray Yildiz ${ }^{* \dagger}$ \\ Mechanical Engineering \\ Bilkent University \\ Ankara, 06800, Turkey \\ Email: yyildiz@bilkent.edu.tr
}

\begin{abstract}
Model reference adaptive control (MRAC) can effectively handle various challenges of the real world control problems including exogenous disturbances, system uncertainties, and degraded modes of operations. In human-in-the-loop settings, MRAC may cause unstable system trajectories. Basing on our recent work on the stability of MRAC-human dynamics, here we follow an optimization based computations to design a linear filter and study whether or not this filter inserted between the human model and MRAC could help remove such instabilities, and potentially improve performance. To this end, we present a mathematical approach to study how the error dynamics of MRAC could favorably or detrimentally influence human operator's error dynamics in performing a certain task. An illustrative numerical example concludes the study.
\end{abstract}

*Professor Yucelen's research was supported in part by the National Aeronautics and Space Administration under Grant NNX15AM51A. Professor Sipahi's research was supported in part by Northeastern University College of Engineering Faculty Fellow Award.

$\dagger$ Address all correspondence to this author.

\section{NOMENCLATURE}

$A, B, C, D, E$ State vector coefficients with their corresponding subscripts

$F_{1,2} \quad$ Filter scalar time constants

$G_{f} \quad$ Linear filter transfer function

$G_{h, f} \quad$ Human-filter transfer function

$k_{p} \quad$ Pilot gain

$T_{z, p} \quad$ Pilot scalar time constants

$W \quad$ Unknown weight matrix

$P \quad$ Solution of the Lyapunov Equation

$c(t) \quad$ Filtered command

$e(t) \quad$ System error

$r(t) \quad$ Bounded reference

$u(t) \quad$ Control input

$u_{a}(t) \quad$ Adaptive controller

$u_{n}(t) \quad$ Nominal controller

$x(t) \quad$ Augmented state vector of integrator and accessible state

$x_{c}(t) \quad$ Integrator state

$x_{p}(t) \quad$ Accessible state vector

$x_{r}(t) \quad$ Reference state vector 
$\xi(t) \quad$ Human-filter state vector

$\tau \quad$ Internal human time-delay

$\theta(t) \quad$ Input to human dynamics

$\Lambda \quad$ Unknown control effective matrix

$\delta_{p} \quad$ Uncertainty

$\sigma \quad$ Known basis function

$\gamma \quad$ Learning rate

$\phi(t) \quad$ Augmented state of reference and human-filter

$\varphi(\cdot) \quad$ non-linear forcing term

\section{INTRODUCTION}

Model reference adaptive controller (MRAC) can effectively cope with system uncertainties arising from ideal assumptions (e.g., linearization, model order reduction, exogenous disturbances, and degraded modes of operations), but the capabilities of MRAC when interfaced with human operators can sometimes be limited. Indeed, in certain applications, when humans are in the loop [1-4], the arising closed loop with MRAC can become unstable. As a matter of fact, such problems are not only limited to MRAC-human interactions and have been reported to arise in various human-in-the-loop control problems including, for example, pilot-induced oscillations [5]. To address these issues, novel control design ideas were proposed and/or experimentally tested including adaptive control as well as smart-cue/smart-gain concepts $[5,6]$.

An analytical framework however aimed at understanding human-induced instability phenomenon and that can ultimately be used to drive rigorous control design is currently lacking. A recent study of the authors aimed exactly at addressing this bottleneck [7]. The cited study developed comprehensive models from a system level perspective and analyzed these models to develop an understanding of stability limits, in particular within the framework of human-in-the-loop MRAC architectures. One key message in [7] was that human reaction delays posed significant limitations on system performance and stability; see also [8] on the stability analysis of time delay systems.

Considering the detrimental effects of time delays as a major problem in human-in-the-loop systems, it is of strong interest to address this problem within the MRAC framework. For this purpose, here we propose to insert a linear filter in between the human model and MRAC, to be designed strategically via optimization-based tools with the aim to enhance both stability and performance characteristics of the combined MRAC-humanfilter closed-loop dynamics. We find that the proposed filter can effectively increase stability limits of the overall closed-loop system. Moreover, the coupling between MRAC and the human model creates an interesting competition, which must be carefully studied for the overall synergistic collaboration between MRAC and the human. To this end, we present a mathematical development to investigate how the error dynamics of MRAC

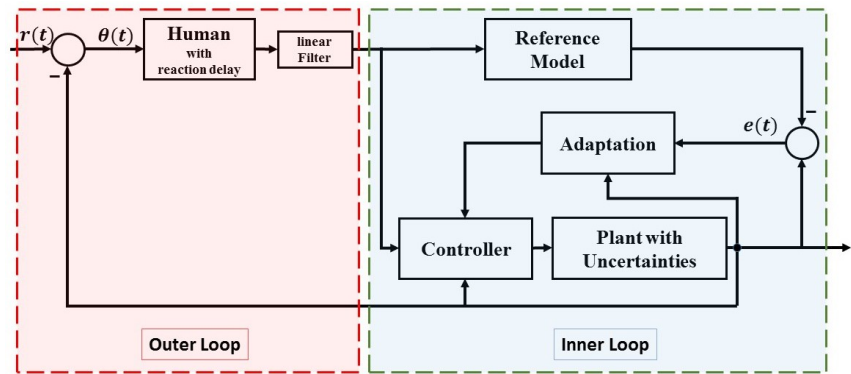

FIGURE 1. Block diagram of the human-in-the-loop model reference adaptive control architecture.

could affect the error dynamics arising in the response of human while trying to achieve a certain task, e.g., step tracking. Our study shows that the proposed filter can be also useful in this relationship with more than an order of magnitude reduction at the critical frequency of the incoming error dynamics.

The article is organized as follows. In Section Problem Formulation, we provide the main discussions regarding problem formulation, including human-MRAC-filter model analysis. In Section Stability in the Presence of Delay, we discuss the stability of the proposed model; then, we study human error-MRAC error relationship. Finally, Section Illustrative Numerical Example concludes the study by providing the reader with numerical illustrations of the discussions.

\section{PROBLEM FORMULATION}

To study human-in-the-loop model reference adaptive controllers, we start with the block diagram configuration given by Fig. 1. In the figure, the outer loop architecture includes the reference that is fed into the human dynamics to generate a command for the inner loop architecture in response to the variations resulting from the uncertain dynamical system. In this setting, the reference input is what the human aims to achieve in a task, and the uncertain dynamical system is the machine on which this task is being performed. The inner loop architecture includes the uncertain dynamical system as well as the model reference adaptive controller components (i.e., the reference model, the parameter adjustment mechanism, and the controller). Specifically, at the outer loop architecture, we consider a general class of linear human models with constant time-delay followed by a linear filter, where the combined human model and linear filter is given by

$$
\begin{aligned}
& \dot{\xi}(t)=A_{\mathrm{h}} \xi(t)+B_{\mathrm{h}} \theta(t-\tau), \quad \xi(0)=\xi_{0}, \\
& c(t)=C_{\mathrm{h}} \xi(t)+D_{\mathrm{h}} \theta(t-\tau),
\end{aligned}
$$

where $\xi(t) \in \mathbb{R}^{n_{\xi}}$ is the internal human-filter state vector, $\tau \in \mathbb{R}_{+}$ is the internal human time-delay, $A_{h} \in \mathbb{R}^{n_{\xi} \times n_{\xi}}, B_{h} \in \mathbb{R}^{n_{\xi} \times n_{r}}, C_{h} \in$ 
$\mathbb{R}^{n_{c} \times n_{\xi}}, D_{h} \in \mathbb{R}^{n_{c} \times n_{r}}$, and $c(t) \in \mathbb{R}^{n_{c}}$ is the filtered command, which is the input to the inner loop architecture as shown in Fig. 1. Here, input to the human dynamics is given by

$$
\theta(t) \triangleq r(t)-E_{h} x(t)
$$

where $\theta(t) \in \mathbb{R}^{n_{r}}$, with $r(t) \in \mathbb{R}^{n_{r}}$ being the bounded reference. Here, $x(t) \in \mathbb{R}^{n}$ is the state vector (further details below) and $E_{h} \in \mathbb{R}^{n_{r} \times n}$ selects the appropriate states to be compared with $r(t)$. Note that this dynamics encompasses the human models with linear time-invariant dynamics with reaction time-delay like Neal-Schmith model [1,2,9-11] and McRuer's model [12].

Next, we summarize from [7]. At the inner loop architecture, we consider the uncertain dynamical system given by

$$
\dot{x}_{p}(t)=A_{p} x_{p}(t)+B_{p} \Lambda u(t)+B_{p} \delta_{p}\left(x_{p}(t)\right), \quad x_{p}(0)=x_{p_{0}},
$$

where $x_{p}(t) \in \mathbb{R}^{n_{p}}$ is the accessible state vector, $u(t) \in \mathbb{R}^{m}$ is the control input, $\delta_{p}: \mathbb{R}^{n_{p}} \rightarrow \mathbb{R}^{m}$ is an uncertainty, $A_{p} \in \mathbb{R}^{n_{p} \times n_{p}}$ is a known system matrix, $B_{p} \in \mathbb{R}^{n_{p} \times m}$ is a known control input matrix, and $\Lambda \in \mathbb{R}_{+}^{m \times m} \cap \mathbb{D}^{m \times m}$ is an unknown control effectiveness matrix where $\mathbb{D}^{m \times m}$ denotes the $n \times n$ real matrices with diagonal scalar entries. Furthermore, we assume that the pair $\left(A_{p}, B_{p}\right)$ is controllable and the uncertainty is parameterized as

$$
\delta_{p}\left(x_{p}\right)=W_{p}^{T} \sigma_{p}\left(x_{p}\right), \quad x_{p} \in \mathbb{R}^{n_{p}}
$$

where $W_{p} \in \mathbb{R}^{s \times m}$ is an unknown weight matrix and $\sigma_{p}$ : $\mathbb{R}^{n_{p}} \rightarrow \mathbb{R}^{s}$ is a known basis function of the form $\sigma_{p}\left(x_{p}\right)=$ $\left[\sigma_{p_{1}}\left(x_{p}\right), \sigma_{p_{2}}\left(x_{p}\right), \ldots, \sigma_{p_{s}}\left(x_{p}\right)\right]^{T}$. To address command following at the inner loop architecture, let $x_{c}(t) \in \mathbb{R}^{n_{c}}$ be the integrator state satisfying

$$
\dot{x}_{c}(t)=E_{p} x_{p}(t)-c(t), \quad x_{c}(0)=x_{c_{0}},
$$

where $E_{p} \in \mathbb{R}^{n_{c} \times n_{p}}$ allows to choose a subset of $x_{p}(t)$ to follow $c(t)$.

Remark 1. Leaving the details to [7], one key contribution from the cited study is that we do not need to make any a-priori assumptions on the boundedness of $c(t)$.

Now, Eq.(4) can be augmented with (6) as

$$
\dot{x}(t)=A x(t)+B \Lambda u(t)+B W_{p}^{T} \sigma_{p}\left(x_{p}(t)\right)+B_{r} c(t)
$$

with $x(0)=x_{0}$, and where

$$
\begin{aligned}
A & \triangleq\left[\begin{array}{ll}
A_{p} & 0_{n_{p} \times n_{c}} \\
E_{p} & 0_{n_{c} \times n_{c}}
\end{array}\right] \in \mathbb{R}^{n \times n}, \\
B & \triangleq\left[B_{p}^{T}, 0_{n_{c} \times m}^{T}\right]^{T} \in \mathbb{R}^{n \times m}, \\
B_{r} & \triangleq\left[0_{n_{p} \times n_{c}}^{T},-I_{n_{c} \times n_{c}}\right]^{T} \in \mathbb{R}^{n \times n_{c}} .
\end{aligned}
$$

and $x(t) \triangleq\left[x_{p}^{T}(t), x_{c}^{T}(t)\right]^{T} \in \mathbb{R}^{n}$ is the augmented state vector, $x_{0} \triangleq\left[x_{p_{0}}^{T}, x_{c_{0}}^{T}\right]^{T} \in \mathbb{R}^{n}$, and $n=n_{p}+n_{c}$. In this inner loop architecture setting, it is practically reasonable to set $E_{h}=\left[E_{h_{P}}, 0_{n_{r} \times n_{c}}\right]$, $E_{h_{p}} \in \mathbb{R}^{n_{r} \times n_{p}}$, in Eq.(3) without loss of theoretical generality since a subset of the accessible state vector is usually available and/or sensed by the human at the outer loop (but not the states of the integrator).

Finally, consider the feedback control law at the inner loop architecture given by

$$
u(t)=u_{n}(t)+u_{a}(t)
$$

where $u_{n}(t) \in \mathbb{R}^{m}$ and $u_{a}(t) \in \mathbb{R}^{m}$ are the nominal and adaptive control laws, respectively. Furthermore, let the nominal control law be

$$
u_{n}(t)=-K x(t)
$$

with $K \in \mathbb{R}^{m \times n}$, such that $A_{r} \triangleq A-B K$ is Hurwitz. For instance, such $K$ exists if and only if $(A, B)$ is a controllable pair. Using Eq.(11) and Eq.(12) in Eq.(7) next yields

$$
\dot{x}(t)=A_{r} x(t)+B_{r} c(t)+B \Lambda\left[u_{a}(t)+W^{T} \sigma(x(t))\right],
$$

where $W^{T} \triangleq\left[\Lambda^{-1} W_{p}^{T},\left(\Lambda^{-1}-I_{m \times m}\right) K\right] \in \mathbb{R}^{(s+n) \times m} \quad$ is an unknown aggregated weight matrix and $\sigma^{T}(x(t))$ $\triangleq\left[\sigma_{p}^{T}\left(x_{p}(t)\right), x^{T}(t)\right] \in \mathbb{R}^{s+n}$ is a known aggregated basis function. Considering Eq.(13), let the adaptive control law be

$$
u_{a}(t)=-\hat{W}^{T}(t) \sigma(x(t))
$$

where $\hat{W}(t) \in \mathbb{R}^{(s+n) \times m}$ is the estimate of $W$ satisfying the parameter adjustment mechanism

$$
\dot{\hat{W}}(t)=\gamma \sigma(x(t)) e^{T}(t) P B, \quad \hat{W}(0)=\hat{W}_{0},
$$

where $\gamma \in \mathbb{R}_{+}$is the learning rate, and system error reads,

$$
e(t) \triangleq x(t)-x_{r}(t)
$$


with $x_{r}(t) \in \mathbb{R}^{n}$ being the reference state vector satisfying the reference system

$$
\dot{x}_{r}(t)=A_{r} x_{r}(t)+B_{r} c(t), \quad x_{r}(0)=x_{r_{0}},
$$

and $P \in \mathbb{R}_{+}^{n \times n} \cap \mathbb{S}^{n \times n}$ is a solution of the Lyapunov equation

$$
0=A_{r}^{T} P+P A_{r}+R
$$

with $R \in \mathbb{R}_{+}^{n \times n} \cap \mathbb{S}^{n \times n}$. Since $A_{r}$ is Hurwitz, it follows from [13] that there exists a unique $P \in \mathbb{R}_{+}^{n \times n} \cap \mathbb{S}^{n \times n}$ satisfying Eq. (18) for a given $R=R^{T}>0 \in \mathbb{R}_{+}^{n \times n} \cap \mathbb{S}^{n \times n}$ where $\mathbb{S}^{n \times n}$ denotes the set of $n \times n$ symmetric matrices.

Remark 2. Here we consider a specific yet widely studied parameter adjustment mechanism given by Eq.(15) and needless to say, one can also consider other types of parameter adjustment mechanisms [14-27] without changing the essence of this paper. For the cases where the basis function $\sigma(\cdot)$ is unknown, extensions follow readily (see, for example, [28]).

\section{STABILITY IN THE PRESENCE OF DELAY}

Stability analysis results of the above developed model can be adapted from our recent study [7]. With the addition of the filter dynamics, to analyze the stability of the coupled inner and outer loop architectures introduced in the previous section, we first write the system error dynamics using Eq.(13), Eq.(14), and Eq.(17) as

$$
\dot{e}(t)=A_{r} e(t)-B \Lambda \tilde{W}^{T}(t) \sigma(x(t)), \quad e(0)=e_{0},
$$

where

$$
\tilde{W}(t) \triangleq \hat{W}(t)-W \in \mathbb{R}^{(s+n) \times m}
$$

is the weight error and $e_{0} \triangleq x_{0}-x_{r_{0}}$. In addition, we write the weight error dynamics using Eq.(15) as

$$
\dot{\tilde{W}}(t)=\gamma \sigma(x(t)) e^{T}(t) P B, \quad \tilde{W}(0)=\tilde{W}_{0},
$$

where $\tilde{W}_{0} \triangleq \hat{W}(0)-W$. The following lemma is now immediate.

Lemma 1. [7] Consider the uncertain dynamical system given by Eq.(4) subject to Eq.(5), the reference model given by Eq.(17), and the feedback control law given by Eq.(11), Eq.(12), Eq.(14), and Eq.(15). Then, the solution $(e(t), \tilde{W}(t))$ is Lyapunov stable for all $\left(e_{0}, \tilde{W}_{0}\right) \in \mathbb{R}^{n} \times \mathbb{R}^{(s+n) \times m}$ and $t \in \overline{\mathbb{R}}_{+}$.
Since the solution $(e(t), \tilde{W}(t))$ is Lyapunov stable for all $\left(e_{0}, \tilde{W}_{0}\right) \in \mathbb{R}^{n} \times \mathbb{R}^{(s+n) \times m}$ and $t \in \overline{\mathbb{R}}_{+}$from Lemma 1 , this trivially implies that $e(t) \in \mathcal{L}_{\infty}$ and $\tilde{W}(t) \in \mathcal{L}_{\infty}$. At this stage in our analysis, it should be noted that one cannot use the Barbalat's lemma [29] to conclude $\lim _{t \rightarrow \infty} e(t)=0$, since $x_{r}(t)$ can be unbounded due to the coupling between the inner and outer loop architectures. Motivated from this standpoint, we next provide the conditions to ensure the boundedness of the reference model states $x_{r}(t)$, which also reveal conditions for stability.

\section{STABILITY ANALYSIS}

Using Eq.(2) in Eq.(17), we write

$$
\begin{aligned}
\dot{x}_{r}(t)= & A_{r} x_{r}(t)+B_{r}\left(C_{h} \xi(t)+D_{h} \theta(t-\tau)\right) \\
= & A_{r} x_{r}(t)-B_{r} D_{h} E_{h} x_{r}(t-\tau)+B_{r} C_{h} \xi(t) \\
& -B_{r} D_{h} E_{h} e(t-\tau)+B_{r} D_{h} r(t-\tau)
\end{aligned}
$$

Next, it follows from Eq.(1) that

$$
\dot{\xi}(t)=A_{h} \xi(t)-B_{h} E_{h} x_{r}(t-\tau)-B_{h} E_{h} e(t-\tau)+B_{h} r(t-\tau) .
$$

Finally, by letting $\phi(t) \triangleq\left[x_{r}^{T}(t), \xi^{T}(t)\right]^{T}$, one can write

$$
\dot{\phi}(t)=\mathcal{A}_{0} \phi(t)+\mathcal{A}_{\tau} \phi(t-\tau)+\varphi(\cdot), \quad \phi(0)=\phi_{0}
$$

where

$$
\begin{aligned}
& \mathcal{A}_{0} \triangleq {\left[\begin{array}{cc}
A_{r} & B_{r} C_{h} \\
0_{n_{\xi} \times n} & A_{h}
\end{array}\right] \in \mathbb{R}^{\left(n+n_{\xi}\right) \times\left(n+n_{\xi}\right)}, } \\
& \mathcal{A}_{\tau} \triangleq\left[\begin{array}{cc}
-B_{r} D_{h} E_{h} & 0_{n \times n_{\xi}} \\
-B_{h} E_{h} & 0_{n_{\xi} \times n_{\xi}}
\end{array}\right] \in \mathbb{R}^{\left(n+n_{\xi}\right) \times\left(n+n_{\xi}\right)}, \\
& \varphi(\cdot) \triangleq\left[\begin{array}{c}
-B_{r} D_{h} E_{h} e(t-\tau)+B_{r} D_{h} r(t-\tau) \\
-B_{h} E_{h} e(t-\tau)+B_{h} r(t-\tau)
\end{array}\right] \in \mathbb{R}^{n+n_{\xi}} .
\end{aligned}
$$

We next provide the following lemma for the system in Eq.(24).

Lemma 2. [7] Consider the following system dynamics given by

$$
\dot{z}(t)=F z(t)+G z(t-\tau)+h(t, z(t)), \quad z(0)=z_{0},
$$

where $z(t) \in \mathbb{R}^{n}$ is the state vector, $F \in \mathbb{R}^{n x n}$ and $G \in \mathbb{R}^{n x n}$ are constant matrices, $\tau$ is the time delay and $h(t, z(t))$ is piecewise constant and bounded nonlinear forcing term, which is in general 
a function of state $z$. If the homogeneous dynamical system given by

$$
\dot{z}(t)=F z(t)+G z(t-\tau)
$$

is asymptotically stable, then the states of the original inhomogeneous dynamical system given by Eq.(28) and hence by Eq.(24) remains bounded for all times.

With Lemma 2, one can now state the following result, which provides a stability condition for the overall human-inthe-loop system and convergence of the system error, $e(t)$, to zero.

Theorem 1. [7] Consider the uncertain dynamical system given by Eq.(4) subject to Eq.(5), the reference model given by Eq.(17), the feedback control law given by Eq.(11), Eq.(12), Eq.(14), and Eq.(15), and the human dynamics given by Eq.(1), Eq.(2), and Eq.(3). Then, $e(t) \in \mathcal{L}_{\infty}$ and $\tilde{W}(t) \in \mathcal{L}_{\infty}$. If, in addition, the real parts of all the infinitely many roots of the following characteristic equation

$$
\operatorname{det}\left(s I-\left(\mathcal{A}_{0}+\mathcal{A}_{\tau} e^{-\tau s}\right)\right)=0
$$

have strictly negative real parts, then $x_{r}(t) \in \mathcal{L}_{\infty}, \xi(t) \in \mathcal{L}_{\infty}$, and $\lim _{t \rightarrow \infty} e(t)=0$.

Several methods can be utilized to study the root locations of Eq.(30) for a given delay $\tau$. The four widely used methods are TRACE-DDE [30], DDE-BIFTOOL [31], QPMR [32], and Lambert-W function [33]. In essence, one provides the matrices $\mathcal{A}_{0}$ and $\mathcal{A}_{\tau}$ as well as the delay $\tau$ to these methods, which then return the numerical values of the rightmost root locations of Eq.(30). If the real part of the rightmost root is negative, $\mathrm{RMP}<0$; then, the system is stable, otherwise unstable $(\mathrm{RMP}>0)$. In the illustrative numerical example provided below, we employ TRACE-DDE readily available for download at https://users.dimi.uniud.it/ dimitri.breda/research/software/

Lemma 3. Consider the control error $e(t)$ in Eq.(16) with Laplace transform $\mathcal{E}(s)$, and $r(t)$ with Laplace transform $R(s)$ as the reference input. Then, the human error $\theta(t)$ in Eq.(3) is determined in Laplace domain by

$$
\Theta(s)=\left(I+E_{h} G_{1}\right)^{-1} R(s)-\left(I+E_{h} G_{1}\right)^{-1} E_{h} \mathcal{E}(s),
$$

where

$$
G_{1} \triangleq\left(s I-A_{r}\right)^{-1}\left(B_{r} C_{h}\left(s I-A_{h}\right)^{-1} B_{h}+B_{r} D_{h}\right) e^{-\tau s}
$$

Proof. Considering the human dynamics given by Eq.(1) and Eq.(2), and the reference model dynamics given by Eq.(17), one can write

$$
X_{r}(s)=\left(s I-A_{r}\right)^{-1} B_{r}\left(C_{h} \xi(s)+D_{h} e^{-\tau s} \Theta(s)\right)
$$

Moreover, notice that, using Eq.(1) we have

$$
\xi(s)=\left(s I-A_{h}\right)^{-1} B_{h} e^{-\tau s} \Theta(s)
$$

Hence, combining Eq.(33) and Eq.(34), the transfer function $G_{1}$ in Eq.(32) follows. Next, with human error defined as

$$
\theta(t)=r(t)-E_{h} x(t)
$$

and, considering the error equation given by (16), we have

$$
\theta(t)=r(t)-E_{h} x_{r}(t)-E_{h} e(t)
$$

By simple manipulations, Eq.(31) follows. $\square$

Notice that the relationship between $\theta(t), r(t)$, and $e(t)$ is important for two reasons. Firstly, it allows to estimate the steady state error in $\theta(t)$ given $r(t)$ whenever the system is stable. Secondly, even if MRAC is properly designed, and its error dynamics $e(t)$ goes to zero in steady state, this dynamics can influence the human error dynamics $\theta(t)$ in an undesirable way. Specifically, certain frequency content in $e(t)$ may excite $\theta(t)$ causing poor performance at the human end.

Based on the given problem formulation, the next section analyzes the stability of the closed-loop system depicted in Fig. 1 for various filter parameters to study the performance of MRAChuman-filter dynamics as well as to better understand the error dynamics $\Theta(s)$ in Eq.(31).

\section{ILLUSTRATIVE NUMERICAL EXAMPLE}

Consider the longitudinal motion of a Boeing 747 airplane linearized at an altitude of $40 \mathrm{kft}$ and a velocity of $774 \mathrm{ft} / \mathrm{sec}$ with the dynamics given by [34]

$$
\dot{x}(t)=A_{p} x(t)+B_{p}\left(u(t)+W^{T} \sigma(x(t)),\right.
$$

where $x(t)=\left[x_{1}(t), x_{2}(t), x_{3}(t), x_{4}(t)\right]^{T}$ is the state vector, and $x(0)=x_{0}$ is the vector of initial conditions. Note that (37) can be equivalently written as (4) with $\Lambda=I$. Here, $x_{1}(t), x_{2}(t)$, and $x_{3}(t)$ respectively represent the components of the velocity along the $x, z$, and $y$ axes of the aircraft with respect to the reference 
axes (in $\mathrm{crad} / \mathrm{sec}$ ), and $x_{4}(t)$ represents the pitch Euler angle of the aircraft body axis with respect to the reference axes (in crad), where 0.01 radian $=1 \mathrm{crad}$ (centiradian). In addition, $u(t) \in \mathbb{R}$ represents the elevator control input (in crad). Finally, $W \in \mathbb{R}^{3}$ is an unknown weighting matrix and $\sigma(x(t))=\left[1, x_{1}(t), x_{2}(t)\right]^{T}$ is a known basis function. In the following simulations, we set $W=\left[\begin{array}{lll}0.1 & 0.3 & -0.3\end{array}\right]^{T}$.

The dynamical system given in (37) is assumed to be controlled using a model reference adaptive controller in Section Problem Formulation. Furthermore, the aircraft is assumed to be operated by a pilot whose Neal-Schmidt Model [9] is given by

$$
k_{p} \frac{T_{z} s+1}{T_{p} s+1} e^{-\tau s}
$$

where $k_{p}$ is the positive scalar pilot gain, $T_{z}$ and $T_{p}$ are positive scalar time constants, and $\tau$ is the pilot reaction time delay assumed to be constant. The values of the parameters used in the simulations are provided in Table 1. Consider next a linear filter

TABLE 1. Numerical data used in illustrative numerical example

\begin{tabular}{|c|c|}
\hline$T_{z}$ & 1 \\
\hline$T_{p}$ & 5 \\
\hline$k_{p}$ & 5 \\
\hline$\tau$ & 0.5 \\
\hline$A_{p}$ & $\begin{array}{cccc}-0.0030 & 0.0390 & 0 & -0.3220 \\
-0.0650 & -0.3190 & 7.7400 & 0 \\
0.0201 & -0.1010 & -0.4290 & 0 \\
0 & 0 & 1 & 0\end{array}$ \\
\hline$B_{p}$ & {$\left[\begin{array}{llll}0.0100 & -0.1800 & -1.1600 & 0\end{array}\right]^{T}$} \\
\hline$E_{p}$ & {$\left[\begin{array}{llll}0 & 0 & 0 & 1\end{array}\right]$} \\
\hline$E_{h}$ & {$\left[\begin{array}{lllll}0 & 0 & 0 & 1 & 0\end{array}\right]$} \\
\hline$B_{r}$ & {$\left[\begin{array}{lllll}0 & 0 & 0 & 0 & 1\end{array}\right]^{T}$} \\
\hline$Q$ & $\operatorname{diag}\left(\left[\begin{array}{lllll}0 & 0 & 0 & 1 & 2.5\end{array}\right]\right)$ \\
\hline
\end{tabular}

of the form

$$
G_{f}=\frac{F_{1} s+1}{F_{2} s+1}
$$

attached in series to the human model, as shown in Fig. 1, where scalars $F_{1}$ and $F_{2}$ are filter time constants. In this case, humanfilter transfer function becomes

$$
G_{h, f}=k_{p} e^{-\tau s} \frac{T_{z} s+1}{T_{p} s+1} \frac{F_{1} s+1}{F_{2} s+1}
$$

which is equivalent to the human-filter state space in Eq.(1) and Eq.(2).

The nominal controller $K$ in Eq.(12) can be obtained via a number of different ways. Here, we utilize a linear quadratic regulator (LQR) approach with the following objective function to be minimized

$$
J(\cdot)=\int_{0}^{\infty}\left(x^{T}(t) Q x(t)+\mu u^{2}(t)\right) d t
$$

where $Q$ is a positive-definite weighting matrix of appropriate dimension as shown in Table 1 , and $\mu$ is a positive weighting scalar. In this setting, the selection of the weighing matrices, as expected, can affect the resulting nominal controller gain $K$, which in turn will determine the reference model dynamics Eq.(17). In the following, the main objective is to study how the filter parameters $F_{1}$ and $F_{2}$ affect the stability of the nominal linear system (Eq.(24) with $\varphi(\cdot)=0$ ) stability with respect to $\mu$, and how $\Theta(s)$ dynamics is governed by MRAC error dynamics $\mathcal{E}(s)$ as discussed in Lemma 3.

Note that the purpose of the numerical examples provided in this section is to understand the effects of filter parameters, without particular emphasis on obtaining enhanced transient response characteristics.

\section{Human-Pilot Dynamics with a Linear Filter}

To study the effects of the filter on the stability of the nominal linear closed-loop system Eq.(24) with $\varphi(\cdot)=0$, we first compute the real part of the rightmost pole (RMP) of this system using TRACE-DDE on the plane of the filter parameters $F_{1}$ and $F_{2}$. Following the discussion of Section Stability Analysis, Fig. 2 depicts the effect of $F_{1}$ and $F_{2}$ on the location of RMP, where only blue areas indicate stability with negative real part of the rightmost pole, $\mathrm{RMP}<0$. In this figure we see that to avoid the boundary of instability when RMP $=0$, a safe choice would be to satisfy $F_{2}>F_{1}$; therefore, a lag compensator is appropriate; see [35] for discussions on compensators.

To decide on the optimal $F_{1}$ and $F_{2}$ values, and explore them in a larger range, Simulated Annealing (SA) method is incorporated next (see, for example, [36-38]). The optimization or energy function for this case is considered to be

$$
J_{S A}=R M P
$$



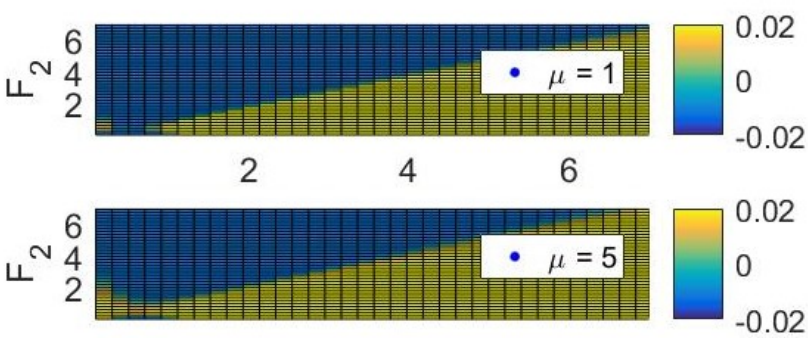

$2 \quad 4$

6
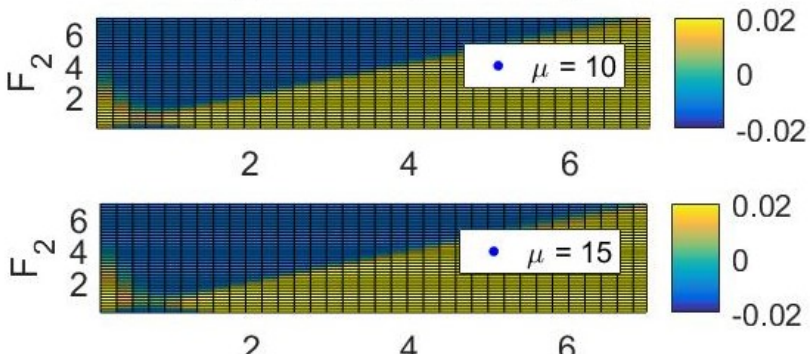

$2 \quad 4 \quad 6$

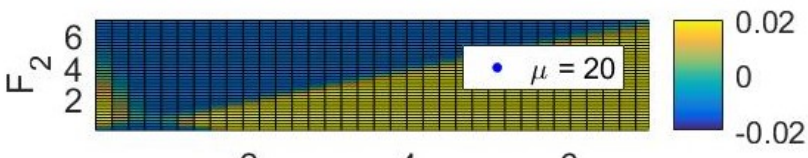

$2 \quad 4 \quad 6$

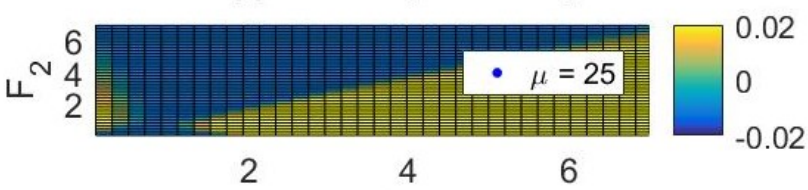

F1

FIGURE 2. Comparison of the effect of $F_{1}$ and $F_{2}$ on the color-coded real part of the rightmost pole (RMP) of the nominal linear system for different penalty gains $\mu$ of LQR. The system is stable for $\mathrm{RMP}<0$, otherwise unstable.

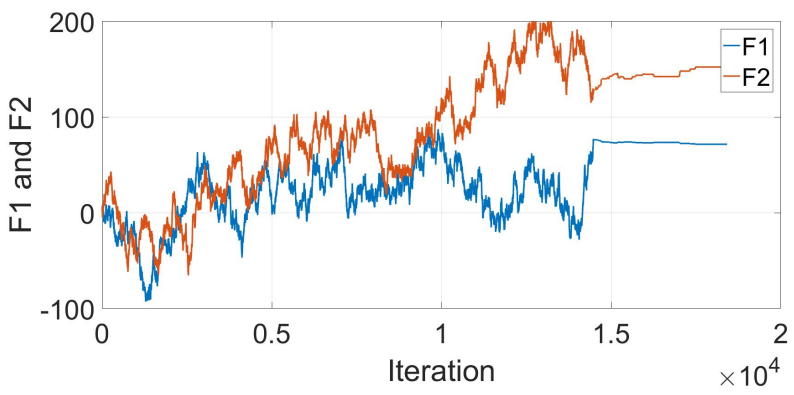

FIGURE 3. $\quad F_{1}$ and $F_{2}$ versus iterations of the Simulated Annealing method.

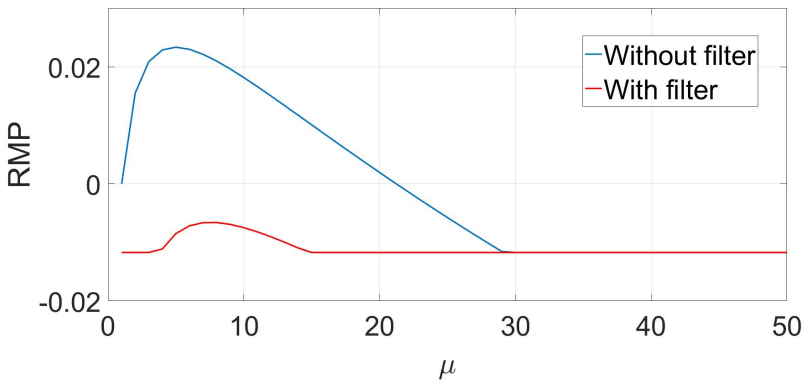

FIGURE 4. The effect of designed linear filter on stability of the linear nominal system with respect to penalty gain $\mu$ of $L Q R$.

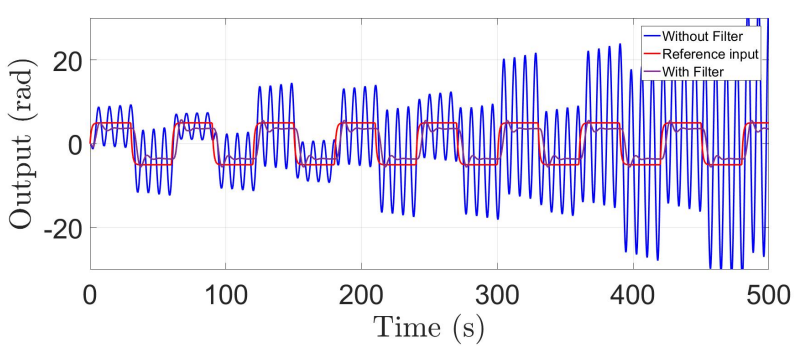

FIGURE 5. Response of the closed-loop nonlinear system with and without using the designed linear filter for $\mu=15$.

as we are concerned with the stability of the system. The method is initialized from the point $F_{1}=F_{2}=1$, which corresponds to the no-filter case. Fig. 3 depicts how Simulated Annealing finds the optimal filter parameters, which are $F_{1}=71.448$ and $F_{2}=152.051$. As the iterations progress, we observe that in most of the steps, $F_{2}>F_{1}$, indicating consistency with the initial findings in Fig. 2. For this filter parameters, we compute $\mathrm{RMP}=-0.012$. One point to note is that in designing the filter parameters using Simulated Annealing, one has to be careful that $A_{r}$ of the reference model remains Hurwitz, otherwise this will violate the conditions of Theorem 1 and will result in instability of the inner loop, and therefore instability of the overall closedloop system. This is the reason why the filter cannot optimize the energy function (42) further especially for higher values of $\mu$ (see Fig. 4).

One key utility of the designed filter is that, with the filter, it is possible to stabilize an unstable MRAC-human closed-loop system. Specifically, considering Fig. 4, one can see that with the value of $\mu=15$ and pilot model settings as in Table 1, the nonlinear closed-loop system is unstable; and, when the linear filter with the parameters obtained by Simulated Annealing method is inserted in the closed-loop system, stability can be recovered. Fig. 5 and its zoom-in version in Fig. 6 depict the time domain 


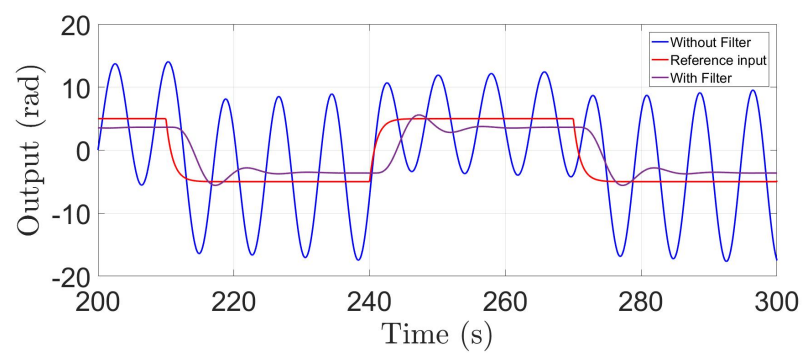

FIGURE 6. Close-up response of the closed-loop nonlinear system obtained in Fig. 5.

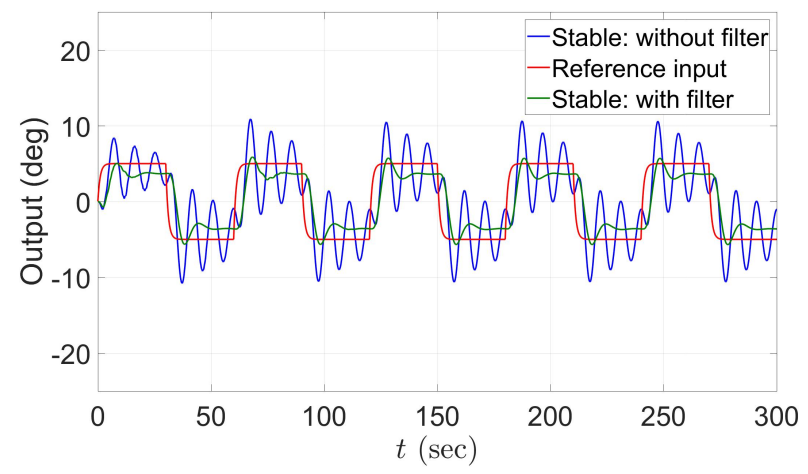

FIGURE 7. Response of the closed-loop nonlinear system with and without using the designed linear filter for $\mu=40$.

response of the system, for both unstable and stabilized systems. ${ }^{1}$

Inspecting Figure 4, it may seem for $\mu>22$ that the filter is ineffective on the stability of the linear nominal system (Eq.(24) with $\varphi(\cdot)=0$ ). However, the presence of the filter improves the transient dynamics, see Fig. 7. Moreover, as previously mentioned, LQR method is used to design the nominal controller $K$ in (12). With $A_{r}=A-B K$, we have that the designed $K$ will determine the reference model dynamics. On the other hand, even if $A_{r}$ is stable, this does not mean the linear nominal system (Eq.(24) with $\varphi(\cdot)=0$ ) is stable. For example, as shown in Fig. 4, for the values of $\mu<20$, the nominal part of Eq.(24) is unstable. Furthermore, as depicted in Figure 8, increasing $\mu$ in the LQR design may not be a feasible option as this will cause larger rise times of the reference dynamics (the inner loop). Consequently, without the proposed filter it is impossible to simultaneously attain faster reference system dynamics and the stability of the linear nominal system. This result clearly demonstrates the utility of the filter.

\footnotetext{
${ }^{1}$ It is worth noting that for the sake of consistency, we selected an unstable case for the without-filter plots, that was stabilized using linear filter.
}

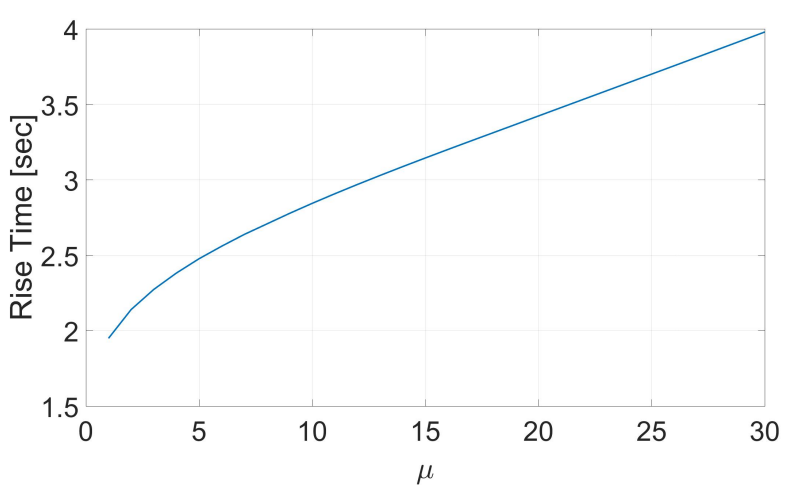

FIGURE 8. Change of the rise time $\left(t_{r}\right)$ of the reference system dynamics (the inner loop) with respect to the penalty gain $\mu$.

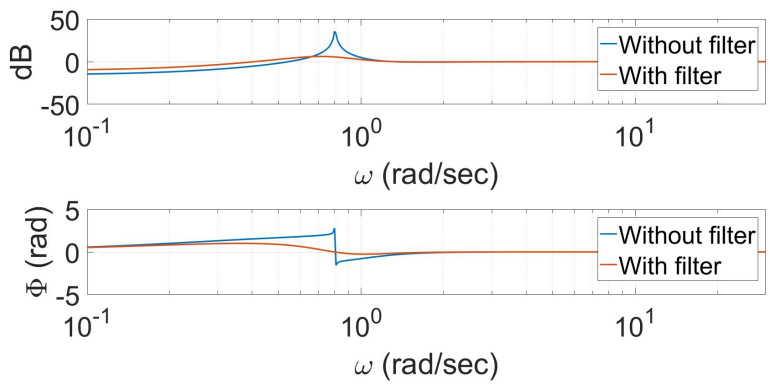

FIGURE 9. Bode plots of the transfer function between the input $\mathcal{E}(s)$ and output $\Theta(s)$ derived in (31) for the case with and without the designed linear filter. Here reference input $R(s)$ is assumed to be zero.

\section{Human error vs. MRAC error}

As discussed in Section Stability Analysis, it is critical to study how human error $\Theta(s)$ is related to the control error signal $\mathcal{E}(s)$. Therefore, we next study the effect of the presence of a linear filter on this relationship. Fig. 9 depicts the Bode plots of the transfer function derived in Eq.(31), assuming $R(s)=0$, for the same pilot model settings as in Table 1. Here, we observe that the filter suppresses undesired peak of $35.854 \mathrm{~dB}$ at $\omega=0.800$ $\mathrm{rad} / \mathrm{sec}$ down to $6.191 \mathrm{~dB}$ at $\omega=0.71 \mathrm{rad} / \mathrm{sec}$, achieving a 26.663 $\mathrm{dB}$ reduction. This indicates that any excitation from MRAC error dynamics $e(t)$ on $\theta(t)$ error of the human at $\omega=0.8 \mathrm{rad} / \mathrm{sec}$ can be reduced more than an order of magnitude, thereby causing much less detrimental effects on the human error dynamics when a lag filter is utilized within the MRAC scheme.

\section{CONCLUSION}

We analyzed human-in-the-loop model reference adaptive control architectures with linear filtering to study the stability conditions and analyze the performance in the presence of hu- 
man reaction delays. Specifically, we designed the filter parameters to stabilize the closed-loop, MRAC-human-filter dynamics. Moreover, a key transfer function between MRAC error dynamics and the human error dynamics arising in the task execution was developed to study how MRAC and human model interact with each other. We showed that the proposed filter was effective in suppressing undesirable oscillations from MRAC dynamics to the human, enabling a more effective and synergistic MRAChuman integration.

\section{REFERENCES}

[1] Ryu, S., and Andrisani, D., 2003. "Longitudinal flying qualities prediction for nonlinear aircraft". Journal of Guidance, Control, and Dynamics, 26(3), pp. 474-482.

[2] Miller, C. J., AIAA Guidance, Navigation, and Control Conference, 2011. "Nonlinear dynamic inversion baseline control law: Architecture and performance predictions".

[3] Trujillo, A., and Gregory, I., 2013. "Adaptive controller adaptation time and available control authority effects on piloting". NASA Technical Reports Server.

[4] Trujillo, A. C., Gregory, I. M., and Hempley, L. E., 2015. "Adaptive state predictor based human operator modeling on longitudinal and lateral control". In AIAA Modeling and Simulation Technologies Conference, p. 0654.

[5] Klyde, D. H., and McRuer, D., 2009. "Smart-cue and smart-gain concepts development to alleviate loss of control". Journal of Guidance, Control, and Dynamics, 32, pp. 1409-1417.

[6] Richards, N. D., Adams, R. J., Klyde, D. H., and Cogan, B., 2015. "Flight-test evaluation of an adaptive controller for flying qualities specification and protection". Journal of Guidance, Control, and Dynamics, 38, pp. 2241-2256.

[7] Yucelen, T., Yildiz, Y., Sipahi, R., Yousefi, E., and Nguyen, N. T., 2017. "Stability analysis of human-adaptive controller interactions". International Journal of Control. in print.

[8] Sipahi, R., Niculescu, S.-I., Abdallah, C. T., Michiels, W., and $\mathrm{Gu}, \mathrm{K} ., 2011$. "Stability and stabilization of systems with time delay". IEEE Control Systems Magazine, 31(1), Feb., pp. $38-65$.

[9] Schmidt, D., and Bacon, B., 1983. “An optimal control approach to pilot/vehicle analysis and the neal-smith criteria”. Journal of Guidance, Control, and Dynamics, 6(5), pp. 339-347.

[10] Thurling, A. J., 2000. Improving uav handling qualities using time delay compensation. Tech. rep., DTIC Document.

[11] Witte, J. B., 2004. An investigation relating longitudinal pilot-induced oscillation tendency rating to describing function predictions for rate-limited actuators. Tech. rep., DTIC Document.
[12] McRuer, D. T., Jan. 1974. "Mathematical models of human pilot behavior".

[13] Haddad, W. M., Chellaboina, V., and Kablar, N. A., December 1999. "Nonlinear impulsive dynamical systems Part I: Stability and dissipativity". In Proc. IEEE Conference on Decision and Control, pp. 4404-4422. Also in International Journal of Control, vol.74, pp.1631-1658, 2001.

[14] Narendra, K. S., and Annaswamy, A. M., 1987. "A new adaptive law for robust adaptation without persistent excitation". IEEE Transactions on Automatic Control, 32(2), pp. 134-145.

[15] Ioannou, P., and Kokotovic, P., 1984. "Instability analysis and improvement of robustness of adaptive control". Automatica, 20(5), pp. 583-594.

[16] Pomet, J. B., and Praly, L., 1992. “Adaptive nonlinear regulation: Estimation from Lyapunov equation". IEEE Transactions on Automatic Control, 37, pp. 729-740.

[17] Yucelen, T., and Calise, A. J., 2010. "Kalman filter modification in adaptive control". Journal of Guidance, Control, and Dynamics, 33(2), pp. 426-439.

[18] Nguyen, N., Krishnakumar, K., and Boskovic, J., Honolulu, Hawaii, 2008. "An optimal control modification to modelreference adaptive control for fast adaptation". Proc. AIAA Guidance, Navigation, and Control Conference.

[19] Nguyen, N., Bakhtiari-Nejad, M., and Ishihira, A., St. Louis, MO, 2010. "Robust adaptive optimal control with large adaptive gain". Proc. American Control Conference.

[20] Yucelen, T., Calise, A. J., and Nguyen, N. T., 2011. "Evaluation of derivative-free adaptive controller with optimal control modification”. In Proceedings of the 2011 AIAA Guidance, Navigation, and Control Conference. Portland, Oregon.

[21] Calise, A. J., and Yucelen, T., 2012. "Adaptive loop transfer recovery". Journal of Guidance, Control, and Dynamics, 35(3), pp. 807-815.

[22] Chowdhary, G., and Johnson, E. N., 2010. "Theory and flight test validation of a concurrent learning adaptive controller". AIAA Journal of Guidance, Control, and Dynamics, 34, pp. 592-607.

[23] Chowdhary, G., Yucelen, T., Mühlegg, M., and Johnson, E. N., 2013. "Concurrent learning adaptive control of linear systems with exponentially convergent bounds". International Journal of Adaptive Control and Signal Processing, 27(4), pp. 280-301.

[24] Yucelen, T., and Calise, A. J., 2011. "Derivative-free model reference adaptive control". Journal of Guidance, Control, and Dynamics, 34(4), pp. 933-950.

[25] Yucelen, T., and Haddad, W. M., 2013. "Low-frequency learning and fast adaptation in model reference adaptive control”. IEEE Transactions on Automatic Control, 58(4), pp. 1080-1085.

[26] Yucelen, T., Gruenwald, B., and Muse, J. A., 2015. "A 
direct uncertainty minimization framework in model reference adaptive control". In AIAA Guidance, Navigation, and Control Conference.

[27] Gruenwald, B., and Yucelen, T., 2015. "On transient performance improvement of adaptive control architectures". International Journal of Control, 88(11), pp. 2305-2315.

[28] Yucelen, T., De La Torre, G., and Johnson, E. N., 2014. "Improving transient performance of adaptive control architectures using frequency-limited system error dynamics". International Journal of Control, 87(11), pp. 23832397.

[29] Khalil, H. K., 1996. Nonlinear Systems. Prentice Hall, Upper Saddle River, NJ.

[30] Breda, D., Maset, S., and Vermiglio, R., 2006. "Pseudospectral differencing methods for characteristic roots of delay differential equations". SIAM Journal of Scientific Computing, 27, pp. 482-495.

[31] Engelborghs, K., Luzyanina, T., and Roose, D., 2000. "Numerical Bifurcation Analysis of Delay Differential Equations Using DDE-BIFTOOL". ACM Transactions on Mathematical Software, 1(1), pp. 1-21.

[32] Vyhlidal, T., and Zitek, P., 2009. "Mapping based algorithm for large-scale computation of quasi-polynomial zeros". IEEE Transactions on Automatic Control, 54(1), pp. 171-177.

[33] Yi, S., Nelson, P. W., and Ulsoy, A. G., 2010. Time-delay Systems: Analysis and Control Using the Lambert W Function. World Scientific Publishing Company.

[34] Bryson, A. E., 1994. Control of Spacecraft and Aircraft. Princeton University Press.

[35] Ogata, K., 2010. Modern Control Engineering. Pearson, Upper Saddle River, NJ.

[36] Eflese, R., 1990. "Simulated annealing: a tool for operational research". European Journal of Operational Research.

[37] Kirkpatrick, S., Gelatt, C. D., and Vecchi, M. P., 1983. "Optimization by simulated annealing”. Science, New Series.

[38] Metrolpolis, N., Rosenbluth, A., Rosenbluth, M., Teller, A. H., and Teller, E., 1953. "Equations of state calculations by fast computing machines". The Journal of Chemical Physics. 